\title{
Study of Tool Wear and Overcut in EDM Process with Rotary Tool and Magnetic Field
}

\author{
Reza Teimouri and Hamid Baseri \\ Mechanical Engineering Department, Babol Noshirvani University of Technology, Babol 47148-71167, Iran \\ Correspondence should be addressed to Hamid Baseri, h.baseri@nit.ac.ir
}

Received 5 December 2011; Accepted 15 January 2012

Academic Editor: Dae Eun Kim

Copyright (๑) 2012 R. Teimouri and H. Baseri. This is an open access article distributed under the Creative Commons Attribution License, which permits unrestricted use, distribution, and reproduction in any medium, provided the original work is properly cited.

Tool wear and workpiece overcut have been studied in electrical discharge machining process with rotational external magnetic field and rotational electrode. Experiments have been divided to three main regimes, namely, low-energy regime, middle-energy regime, and high-energy regime. The influence of process parameters were investigated on electrode wear rate and overcut. Results indicate that applying a magnetic field around the machining gap increases the electrode wear rate and overcut. Also, rotation of the tool has negative effect on overcut.

\section{Introduction}

Materials such as tool steel, ceramic, metal matrix composite, and Inconel widely are used in die molding, aerospace, surgical and automotive industries. Machining of these materials by using traditional machining is difficult due to high wear and corrosion resistance, hardness, and toughness properties of them. Electrical discharge machining (EDM) is a nontraditional method which is used most commonly to machining of difficult-to-machine materials.

Flushing the debris from the machining gap is one of the major challenges in EDM process. Accumulation of debris in gap space causes inactive pulses such as short and open circuit and arc. These types of discharges do not remove any material from the workpiece and damage the surface integrity of machined specimen. By considering these reasons, researchers focus on the methods which improve the flushing in EDM. Methods such as EDM with rotating tool electrode [1-6], ultrasonic vibration assisted EDM [7-10], and compaction of vibration and rotation of electrode $[5,11]$ were introduced to develop the flushing, the eroded particle in gap space and improve the machining performance.

Few researches have investigated the effect of rotary tool on machining characteristics in EDM. Soni and Chakraverti [1] analyzed the effect of rotary electrode tool on the EDM of titanium alloy. They found that the rotary motion of the tool increases the MRR and electrode wear rate (EWR) in all levels of current and pulse on time. Mohan et al. [2, 3] conducted the experimental study on $\mathrm{Al}-\mathrm{SiC}$ composite material. They showed that the rotary electrode improves the MRR and reduce the surface roughness. Kuppan et al. [4] investigated the effects of various rotational speed of electrode on inconel 718. Results show that the increasing of the rotational speed is effective factor in low discharge energy. Ghoreishi and Atkinson [5] studied the influences of vibration and rotation of electrode on machining characteristics in three levels of machining pulse energy. Saha and Choudhury [6] applied the rotation of the electrode on dry EDM.

Magnetic field extensively is used in nontraditional manufacturing process. Magnetic abrasive finishing (MAF) and magnetic abrasive flow finishing (MAFF) are effective methods to producing a mirror surface on metal, ceramic and composite materials [12-15]. In recent years, researchers used the magnetic field in electrolytic finishing process [16]. They found that using magnetic field changes the ion migration paths from linear to cycloid motion. Brujin et al. [17] investigated the effects of magnetic field in gap cleaning and indicated that the magnetic field can improve the gap cleaning process.

Although some researches associated the magnetic field with the manufacturing process, only a few work investigated 


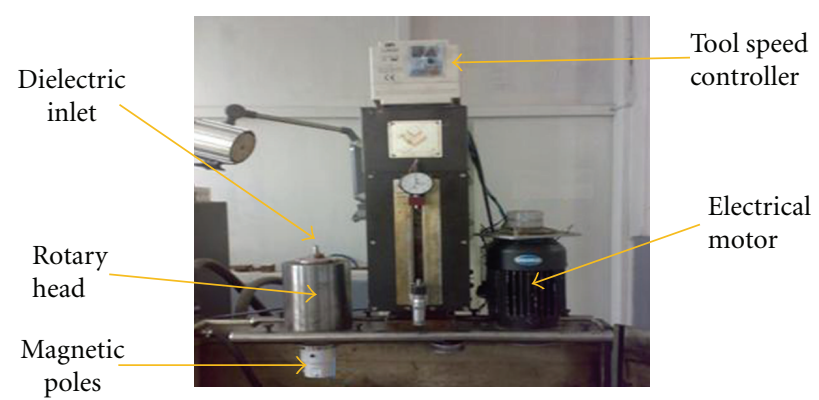

(a)

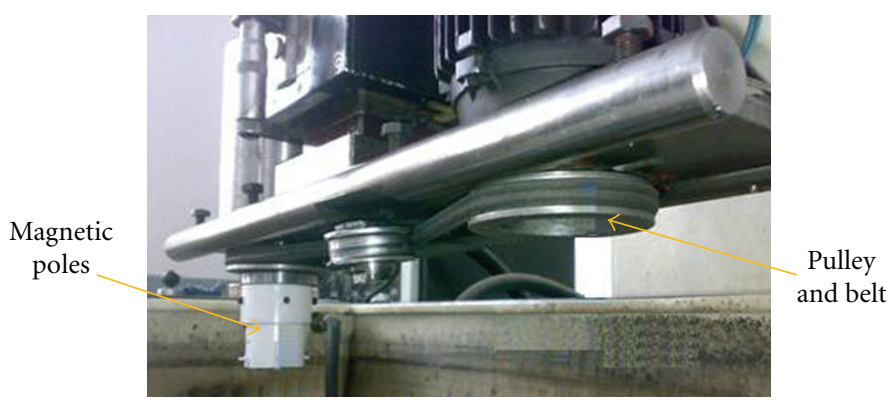

(b)

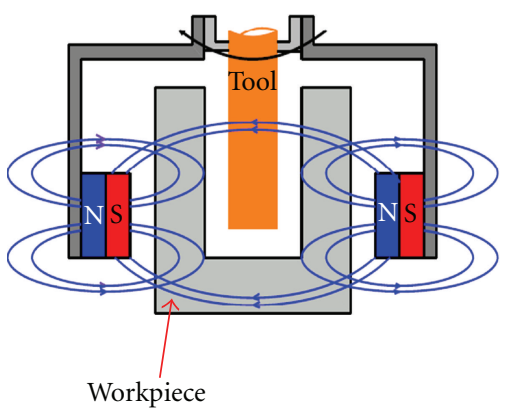

(c)

FIgURE 1: Experimental setup: (a) mounted setup on Tehran Ekram machine, (b) rotation mechanism of the tool and magnetic poles container, (c) schematic diagram of magnetic poles container and magnetic field lines during workpiece.

the effects of magnetic force on EDM performance. Lin and Lee [18] used the magnetic field in EDM process and reported that the magnetic force runs the debris away from machining gap and improves the characteristics of this operation especially in high discharge energy regime. Chattopadhyay et al. [19] used the multilevel intensity of magnetic field and rotary tool electrode with various speed in EDM process and indicated the effects of them on MRR and EWR. Although there are few researches which studied on effects of external magnetic field in EDM performances, the overcut was not studied exactly in published literatures. Also, establishing of external magnetic field on EDM process in fulfilled researches was not focused precisely in machining gap.

In this research, the effects of rotational external magnetic field with various intensities and also various levels of tool rotational speed were studied on EDM performance. The main difference of this work with the others is applying of rotary magnetic field exactly around machining gap in which it aids ionization and formation of plasma channel. Also, the investigated output parameters of the process are EWR and overcut (OC).

\section{Experiments}

2.1. Experimental Setup. In order to find the effects of external magnetic field and rotation of electrode on machining performance, numbers of 39 experimental tests have been carried out on Tehran Ekram 304H/60A die sinking electrical discharge machine with Iso-pulse generator. For rotary-toolassisted tests, an electromotor with a belt-pulley mechanism mounted on machine to generate rotary motion in tool spindle. And tool rotational speed has been controlled by LS600 inverter. Experimental setup was shown at Figures 1(a) and $1(\mathrm{~b})$.

Also, in order to establish an external magnetic field around the tool-workpiece system, two magnetic poles with various intensities ( 0.38 and 1.2 Tesla) were attached on an inner surface of cylinder with central through hole. Figure 1(c) depicts the schematic diagram of magnetic container device and installation of tool and tool holder. Here, magnetic poles revolve around the workpiece by rotation of electrode. Also, magnetic force lines have been demonstrated in this figure.

The machine control unit involves one current meter and voltmeter which was connected in series and parallel, respectively, by spark gap to measure the average current and voltage. Also, in order to monitor and control the process stability, the machine was equipped with two ARCs and short circuit LED which report the gap state.

2.2. Experiment Materials. The workpiece material was SPK cold work steel which is extensively used in dies and mold industry. The specimens were cut to cylinders with $14 \mathrm{~mm}$ diameter and $30 \mathrm{~mm}$ height and then faced and ground with grinding wheel. The chemical compositions of workpiece are listed Table 1. The tool electrode material was also copper with $99.9 \%$ purity, in the size of $10 \mathrm{~mm}$ diameter and $70 \mathrm{~mm}$ 
TABLE 1: Chemical composition of SPK material (X210Cr12).

\begin{tabular}{lccccccccc}
\hline Element & $\mathrm{C}$ & $\mathrm{Si}$ & $\mathrm{Mn}$ & $\mathrm{P}$ & $\mathrm{S}$ & $\mathrm{Cr}$ & $\mathrm{Mo}$ & $\mathrm{Ni}$ & $\mathrm{Co}$ \\
\hline $\mathrm{Wt} \%$ & 2.1 & 0.39 & 0.25 & 0.01 & 0.03 & 11.20 & 0.04 & 0.15 & $<0.005$ \\
\hline
\end{tabular}

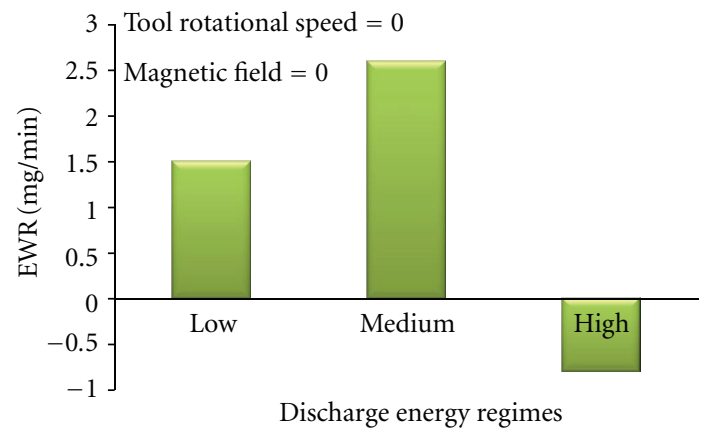

(a)

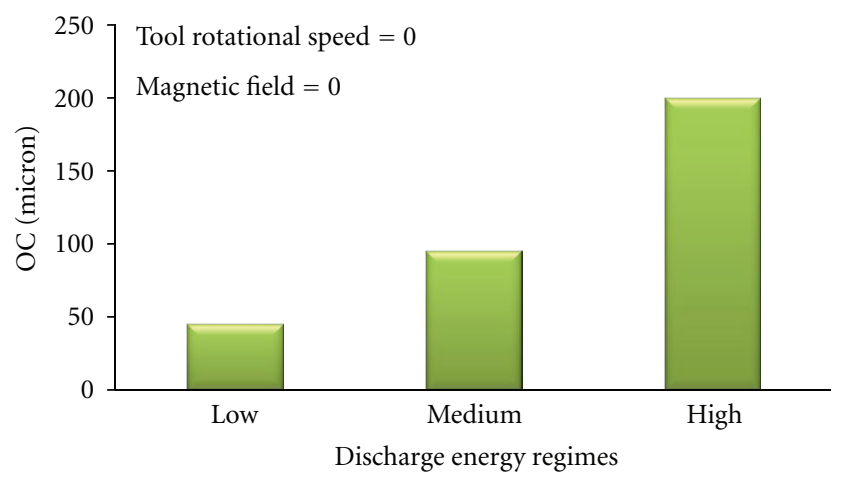

(b)

FIgURE 2: Effects of discharge energy on (a) EWR and (b) OC.

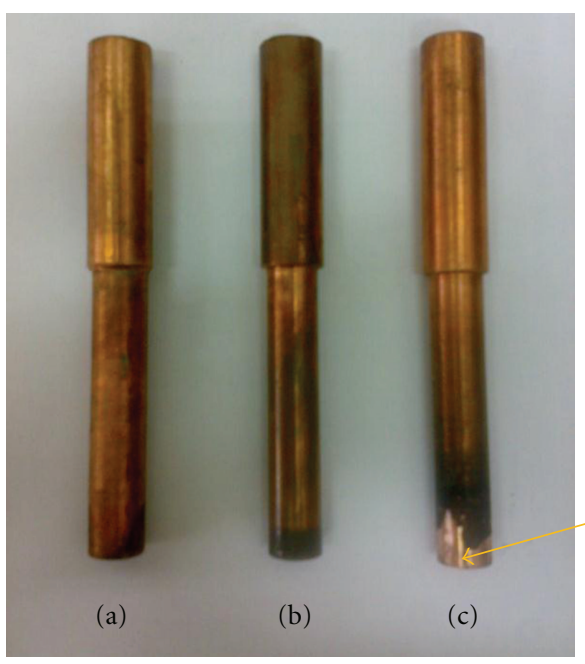

Deposited pyrolytic carbon

FIGURE 3: Electrode surface in (a) low-energy regime, (b) middleenergy regime, (c) high-energy regime which increases the tool weight due to deposited pyrolytic carbon.

height. Table 2 reports the essential physical properties of the copper tool.

2.3. Experimental Procedure. In this work, the experimental procedure has two stages. In the first stage, the effect of three regimes of process parameters on EDM performance is investigated while the electrode is stationary and there is not any magnetic field on machining zone. Three regimes are: (a) low-energy regime, (b) middle-energy regime, and (c) highenergy regime. Variables of these regimes are average current $\left(I_{\mathrm{av}}\right)$, pulse on time $\left(t_{\mathrm{on}}\right)$, and the pulse off time $\left(t_{\text {off }}\right)$ as listed in Table 3. In order to improve experimental condition at
TABLE 2: Main properties of copper electrode.

\begin{tabular}{lc}
\hline Properties & Description \\
\hline Specific gravity $\left(\mathrm{g} / \mathrm{cm}^{3}\right)$ & 8.94 \\
Melting range $\left({ }^{\circ} \mathrm{C}\right)$ & $1065-1083$ \\
Thermal conductivity $(\mathrm{W} / \mathrm{Mk})$ & 388 \\
Specific heat $(\mathrm{J} / \mathrm{kg} \mathrm{K})$ & 385 \\
Thermal expansion coefficient $\left(1 /{ }^{\circ} \mathrm{C}\right)$ & $16.7 \times 10^{-6}$ \\
Electrical resistivity $(\Omega \mathrm{cm})$ & $1.7 \times 10^{-6}$ \\
\hline
\end{tabular}

TABLE 3: EDM setting for each energy regime.

\begin{tabular}{lccc}
\hline Regimes & \multicolumn{3}{c}{ Variables } \\
& $I_{\mathrm{av}}(\mathrm{A})$ & $t_{\mathrm{on}}(\mu \mathrm{s})$ & $t_{\text {off }}(\mu \mathrm{s})$ \\
\hline Low energy & 2.5 & 50 & 25 \\
Middle energy & 9 & 100 & 75 \\
High energy & 21 & 200 & 150 \\
\hline
\end{tabular}

each level of energy, the duty factor must be equal. So by variation of pulse on time, pulse off time must be varied for steady duty factor in all experiments.

In the second stage, the effects of rotational speed of electrode and discharge regime on machining performance are investigated for three state of (i) without external magnetic field, (ii) external magnetic field with low intensity (0.38 Tesla), and (iii) external magnetic field with high intensity (1.2 Tesla). Here, the rotational speeds of the electrode are varied as 0, 200, 500, 800, and 1000 RPM, and experiments were carried out for three regimes.

In order to evaluate the EWR, the WTB RADWAG, an electronic weigh balance with $1 \mathrm{mg}$ resolution, has been used. 


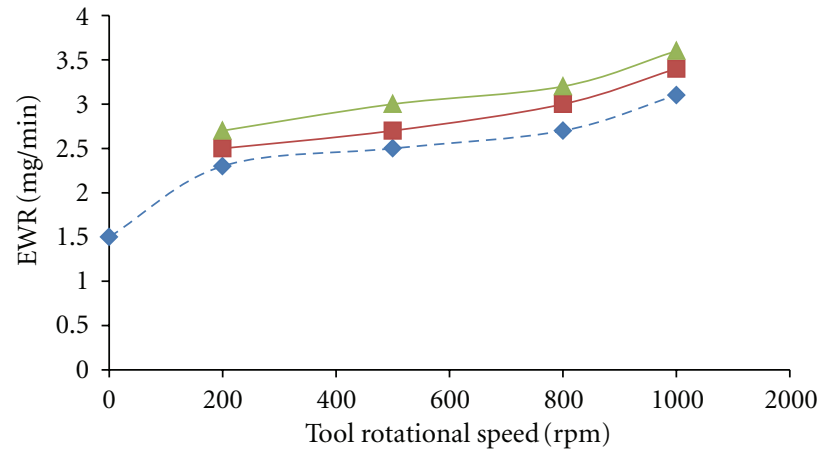

(a) Variation of EWR versus tool rotational speed and magnetic field intensity in low-energy regime

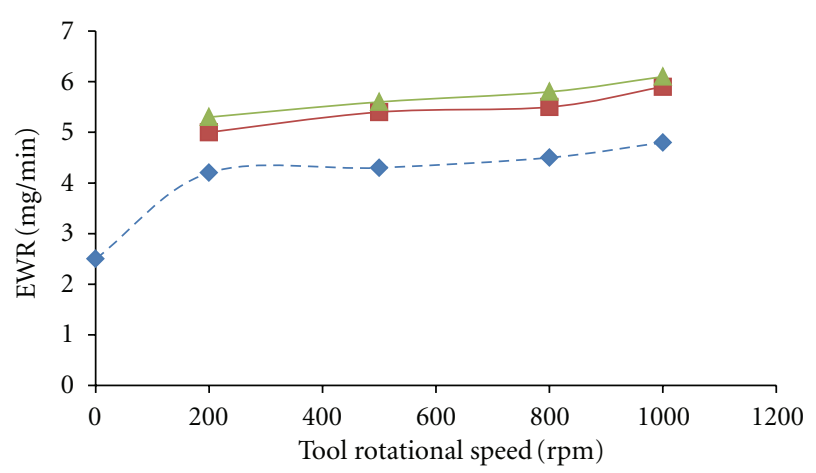

(b) Variation of EWR versus tool rotational speed and magnetic field intensity in middle-energy regime

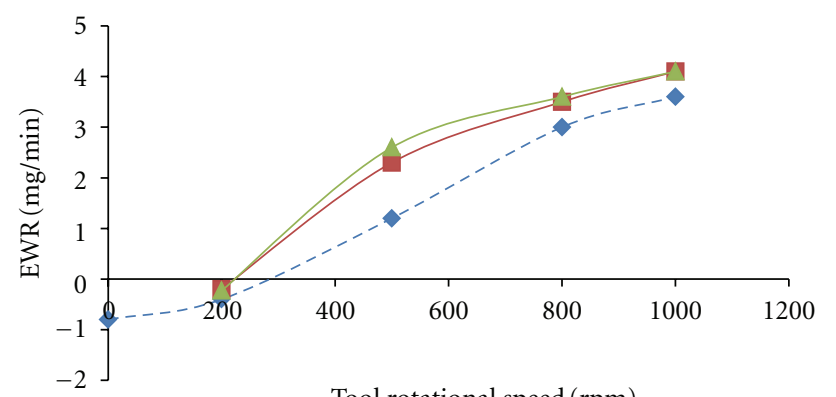

Tool rotational speed (rpm)

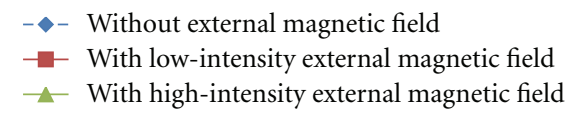

(c) Variation of EWR versus tool rotational speed and magnetic field intensity in high-energy regime

FIGURE 4: EWR versus rotary speed of electrode in different magnetic field for regimes of (a) low energy, (b) middle energy, and (c) high energy.

TABle 4: Description of the electrical discharge machine parameters.

\begin{tabular}{lc}
\hline Parameters & Value \\
\hline No load voltage $(\mathrm{V})$ & 80 \\
Tool polarity & Positive $(+)$ \\
Type of generator & ISO-Pulse \\
Type of flushing & Normal submerged \\
Dielectric & Kerosene \\
Machining time $(\min )$ & 45 \\
\hline
\end{tabular}

Each tool specimen was weighed before and after machining process and EWR determined as follows:

$$
\mathrm{EWR}=\frac{\text { tool mass loss }}{\text { machining time }}(\mathrm{mg} / \mathrm{min})
$$

where the machining time was $45 \mathrm{~min}$ for all experiments.

Due to some instrumentation limits, the overcut could not be studied in all machined depth. So the values of overcut have been obtained by

$$
\mathrm{OC}=R_{2}-R_{1} \text { (micron), }
$$

where $R_{1}$ is radius of tool before machining $(5 \mathrm{~mm})$ and $R_{2}$ is edge radius of hole after machining. Description of the electrical discharge machine parameters in all experiments have listed in Table 4. In order to decrease the experimental error, three specimens have been tested for each kind of conditions.

\section{Results and Discussion}

3.1. Analysis of Discharge Energy Effects on EDM Performance. As discussed in Section 2.3, experiments have been done in two stages. In stage one, the effects of discharge energy on EWR and OC were investigated for stationary electrode and without external magnetic field as shown in Figure 2.

3.1.1. Effect of Discharge Energy on EWR. The discharge energy that is transfered on machining gap is divided between electrode, workpiece, and dielectric fluid in discharge channel. It can be seen from Figure 2(a), that by increasing the discharge energy, the amount of melted and vaporized materials increase and lead to increasing in the EWR. But by observing the EWR at high-energy regime, the negative value is found. This is because of the pyrolytic 


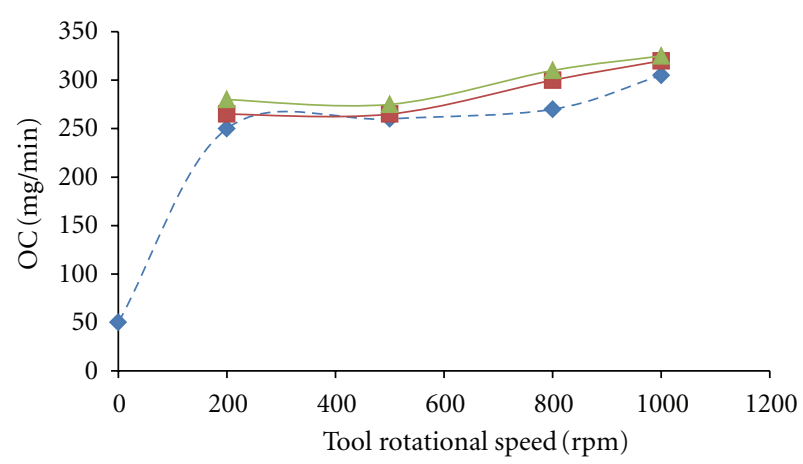

(a) Variation of OC versus tool rotational speed and magnetic field intensity in low-energy regime

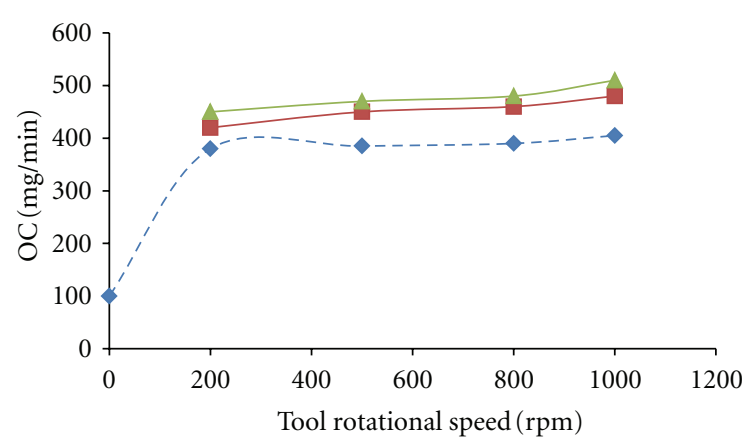

(b) Variation of OC versus tool rotational speed and magnetic field intensity in middle-energy regime

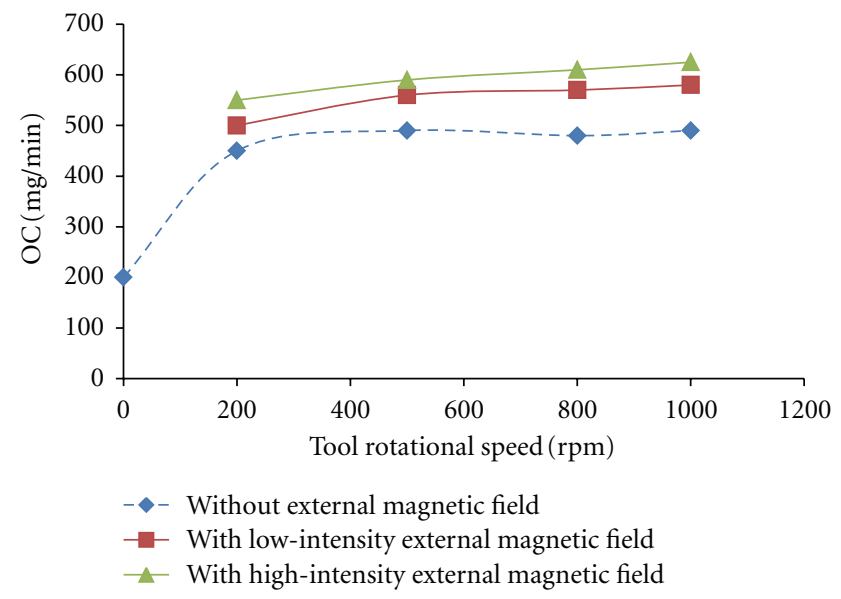

(c) Variation of OC versus tool rotational speed and magnetic field intensity in high-energy regime

FigURE 5: OC versus rotary speed of electrode in different magnetic field for regimes of (a) low energy, (b) middle energy, and (c) high energy.

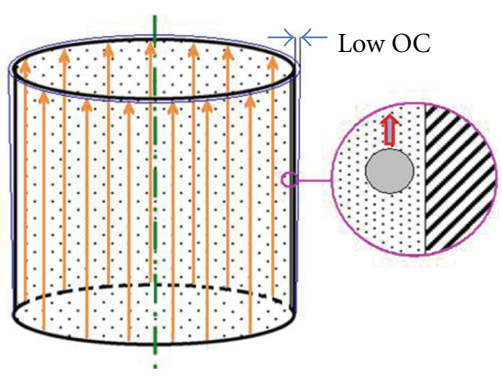

(a)

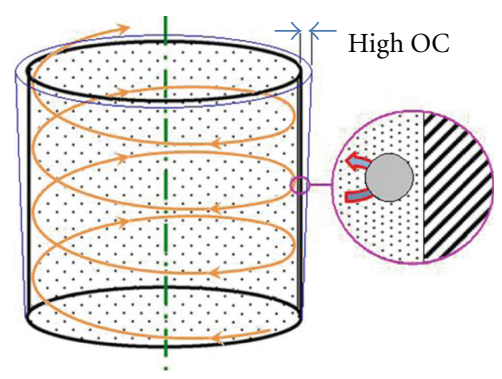

(b)

FIGURE 6: Path of debris motion and OC value for (a) stationary tool without magnetic field, (b) rotary tool and magnetic field.

carbon which is deposited from dielectric fluid in highenergy regime. When the process is in high-energy regime, this energy affects on dielectric medium and deposits it. The pyrolytic carbon is one of the main components that are obtained by deposition of kerosene dielectric. Pyrolytic carbon deposits on the electrode surface, and it acts as a protective layer. Also, it protects the electrode surface against the melting and vaporizing at high discharge energy and therefore decreases the EWR.
Figure 3 shows the amount of pyrolytic carbon which is sited on the electrode surface. It can be seen that the most carbon is in high-energy regime and it is negligible in the lower-energy ones.

3.1.2. Effect of Discharge Energy on OC. According to Figure 2(b), by enhancement in the discharge energy, the overcut will increase due to high value of thermal energy transfer to machining zone. This phenomenon leads to 


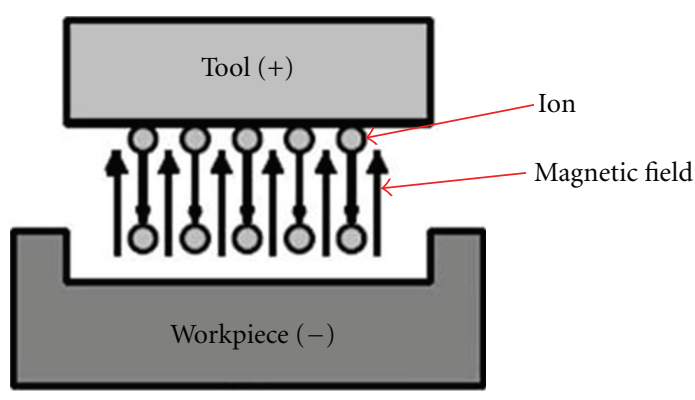

(a)

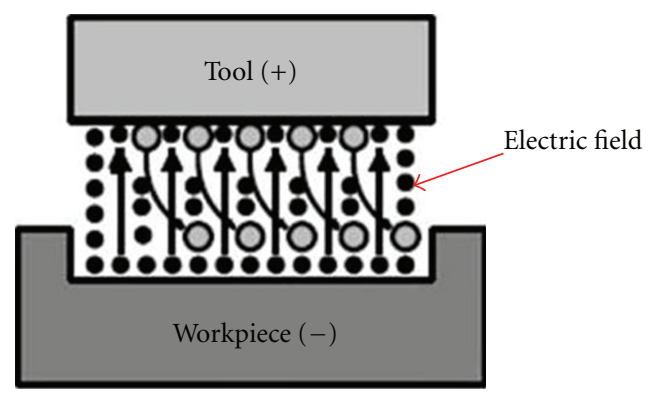

(b)

FIGURE 7: Ion path motion in gap space (a) without external magnetic field, (b) with applying an external magnetic field.

melting and vaporizing more material from workpiece surface. Therefore, the diameter and depth of discharge crater are increased and more overcut occurs at high-energy regime, in comparison of low one.

In the next section, the effects of rotational speed of electrode and discharge regime on machining performance are analyzed while the machining zone has no magnetic field, low-intensity magnetic field (0.38 Tesla), and high-intensity magnetic field (1.2 Tesla).

\subsection{Analysis of Rotational Speed and Magnetic Field Effects on EWR}

3.2.1. Effect of Rotational Speed of Tool Electrode on EWR. According to results of EWR shown in Figure 4, by increasing, the rotational speed of electrode EWR increases at each level of discharge energy. It can be explained as follows; by increasing tool rotary speed, the centrifugal force increases subsequently, so it can throw out the pyrolytic carbon particles from electrode surface and EWR increases. On the other hand, increasing of the suspended debris in machining gap leads to better deposition of the dielectric fluid and decreases the EWR [18]. Thus, each operation which reduces the amount of debris in machining gap may lead to increasing in EWR. Therefore, when the rotary speed increases due to better flushing, the amount of debris in machining gap is declined which leads to increasing in EWR.

3.2.2. Effect of Magnetic Field on EWR. Figure 4 shows that, by using external magnetic field in machining zone, the EWR increases in comparison with situation in which no external magnetic field applied. Because the magnetic field expels the debris from machining gap and improves the process stability it can reduce the inactive pulses. Also, magnetic field aids the ionization and decreases ignition delay time. By considering the mentioned reasons, better transferring of energy leads to more EWR.

Moreover, using magnetic field reduces the amount of debris in machining gap and leads to decrease in amount of pyrolytic carbon which results in higher EWR. Also, Figure 4 shows that there is no difference between EWR results while the magnetic field has low or high intensity.

Although pyrolytic carbon which is formed in the EDM process has thickness, the amount of this thickness is very low and negligible in comparison with amount of overcut induced by process parameters. In other words, thickness of pyrolytic carbon film does not affect overcut due to its negligible value.

\subsection{Analysis of Rotational Speed and Magnetic Field Effects on OC}

3.3.1. Effect of Rotational Speed of Tool Electrode on OC. According to Figure 5, it can be inferred, that by increasing the rotational speed, the overcut increases. This is due to the fact that the centrifugal force increases by increasing the rotational speed, and soit can remove more debris from machining gap. So the removed debris can be placed between the side wall of electrode and workpiece, and spark is occurred between them. This phenomenon leads to increasing the overcut.

3.3.2. Effect of Magnetic Field on OC. Figure 5 shows that, by establishing the magnetic field, overcut will increase. This is due to the fact that, by applying the magnetic field, the number of active pulses increases due to better flushing of machining gap. The active pulses lead to removing more material from workpiece and increasing the size of crater and increasing the over size. Also, the magnetic force acts on machining debris and runs them away from machining gap. When the debris is expelled from machining gap, they are placed between the side walls of tool electrode and workpiece and lead to generating discharge between them, and, as a result, OC increases. Figure 6 shows the path of debris motion for high OC and low OC.

Another reason which leads to increasing overcut will be described as follows: as we all know, the plasma channel consists of electrons and positive-charged ions. Also there is and electrical field in EDM process between tool an workpiece. When a magnetic field is applied vertically around the magnetic field, it can affect charged ions due to Lorentz force (force which arises due to association of magnetic and electric fields). So magnetic field can change the ions path migration and lead to more accident between ions (Figure 7). Therefore, it increases the plasma channel temperature and results in its expansion. High-intensity magnetic field may lead to more change in ion path migration and more expansion of plasma channel. As we all know, in EDM, each 
factor which leads to expansion of plasma channel results in higher OC. The equations that relate to changing the particles in ion path migration are in the appendix.

\section{Conclusion}

In this paper, the influences of rotational electrode and rotational magnetic field with various intensities on EWR and OC are studied. Main conclusions of this research can be summarized as follows.

(1) The electrode wear rate increases by increasing the discharge energy, but, at the high-energy regime, the pyrolytic carbon which is obtained by deposition of dielectric fluid at high energy regime acts as protective layer and reduces the EWR.

(2) Increasing the rotational speed of electrode and magnetic field increases the electrode wear rate due to removing the debris from machining gap which restricts the formation of pyrolytic carbon.

(3) When process moves from low-energy regime to high energy one, the overcut (side gap) increases due to increasing the sizes of discharge crater.

(4) Increasing rotational speed of electrode and establishing magnetic field expel the debris from machining gap and trap them between side walls of workpice and tool electrode and increase the overcut.

(5) The magnetic field causes changing the ion path migration by generating Lorentz force around the electric field, and this force increases the lead overcut.

\section{Appendix}

The moving path of charged particles driven by magnetic field is given by the following equations:

$$
\begin{aligned}
F & =q(E+v \times B), \\
\frac{d v}{d t} & =\frac{q}{m}(E+v \times B),
\end{aligned}
$$

where $m(\mathrm{~g})$ and $q(\mathrm{C})$ represent the mass and charge of ion, respectively. $E\left(\mathrm{Vm}^{-1}\right)$ is the electrical field strength, $B(\mathrm{~T})$ is the magnetic flux density, and $v\left(\mathrm{~ms}^{-1}\right)$ is the ion velocity. As shown in Figure 1(c) the magnetic field that is vertical to electric field changes the path of ionic movement to cycloid curve due to Lorentz force. From (A.3) the equation of motion of the ions is as follows. Assuming $x-y$ coordinate

$$
\begin{gathered}
v_{x}=\frac{V}{B l} \sin \left(\frac{q B}{m} t\right)+v_{x}(0) \cos \left(\frac{q B}{m} t\right), \\
v_{y}=\frac{V}{B l}\left(1-\cos \left(\frac{q B}{m} t\right)\right)+v_{x}(0) \sin \left(\frac{q B}{m} t\right)+v_{y}(0),
\end{gathered}
$$

where $v_{x}$ and $v_{y}$ are the velocity of ions on $x$ and $y$ directions. $v_{x}(0)$ and $v_{y}(0)$ represent the initial velocity of ion on $x$ and $y$ directions. Also, $V$ is the gap voltage and $l$ is gap distance in $(\mathrm{mm})$.
Finally, the path equation of the ions is

$$
\begin{aligned}
x= & \frac{m V}{q B^{2} l}\left(1-\cos \left(\frac{q B}{m} t\right)\right)+\frac{m}{q B} v_{x}(0) \sin \left(\frac{q B}{m} t\right)+x_{0}, \\
y= & -\frac{m V}{q B^{2} l} \sin \left(\frac{q B}{m} t\right)+\frac{m}{q B} v_{x}(0)\left(1-\cos \left(\frac{q B}{m} t\right)\right) \\
& +\left(\frac{V}{B l}+v_{y}(0)\right) t+y_{0},
\end{aligned}
$$

where $x$ and $y$ are the trace of the ion and $x_{0}, y_{0}$ represent the initial position of the ions in electrical discharge regime.

\section{References}

[1] J. S. Soni and G. Chakraverti, "Machining characteristics of titanium with rotary electro-discharge machining," Wear, vol. 171, no. 1-2, pp. 51-58, 1994.

[2] B. Mohan, A. Rajadurai, and K. G. Satyanarayana, "Effect of $\mathrm{SiC}$ and rotation of electrode on electric discharge machining of Al-SiC composite," Journal of Materials Processing Technology, vol. 124, no. 3, pp. 297-304, 2002.

[3] B. Mohan, A. Rajadurai, and K. G. Satyanarayana, "Electric discharge machining of Al-SiC metal matrix composites using rotary tube electrode," Journal of Materials Processing Technology, vol. 153-154, no. 1-3, pp. 978-985, 2004.

[4] P. Kuppan, A. Rajadurai, and S. Narayanan, "Influence of EDM process parameters in deep hole drilling of Inconel 718," International Journal of Advanced Manufacturing Technology, vol. 38, no. 1-2, pp. 74-84, 2008.

[5] M. Ghoreishi and J. Atkinson, "A comparative experimental study of machining characteristics in vibratory, rotary and vibro-rotary electro-discharge machining," Journal of Materials Processing Technology, vol. 120, no. 1-3, pp. 374-384, 2002.

[6] S. K. Saha and S. K. Choudhury, "Experimental investigation and empirical modeling of the dry electric discharge machining process," International Journal of Machine Tools and Manufacture, vol. 49, no. 3-4, pp. 297-308, 2009.

[7] A. Abdullah and M. R. Shabgard, "Effect of ultrasonic vibration of tool on electrical discharge machining of cemented tungsten carbide (WC-Co)," International Journal of Advanced Manufacturing Technology, vol. 38, no. 11-12, pp. 1137-1147, 2008.

[8] Q. H. Zhang, R. Du, J. H. Zhang, and Q. B. Zhang, "An investigation of ultrasonic-assisted electrical discharge machining in gas," International Journal of Machine Tools and Manufacture, vol. 46, no. 12-13, pp. 1582-1588, 2006.

[9] Y. C. Lin, B. H. Yan, and Y. S. Chang, "Machining characteristics of titanium alloy (Ti-6Al-4V) using a combination process of EDM with USM," Journal of Materials Processing Technology, vol. 104, no. 3, pp. 171-177, 2000.

[10] G. S. Prihandana, M. Mahardika, M. Hamdi, Y. S. Wong, and K. Mitsui, "Effect of micro-powder suspension and ultrasonic vibration of dielectric fluid in micro-EDM processes-Taguchi approach," International Journal of Machine Tools and Manufacture, vol. 49, no. 12-13, pp. 1035-1041, 2009.

[11] Z. Y. Yu, Y. Zhang, J. Li, J. Luan, F. Zhao, and D. Guo, "High aspect ratio micro-hole drilling aided with ultrasonic vibration and planetary movement of electrode by micro-EDM," CIRP Annals-Manufacturing Technology, vol. 58, no. 1, pp. 213-216, 2009. 
[12] H. Yamaguchi and T. Shinmura, "Study of an internal magnetic abrasive finishing using a pole rotation system. Discussion of the characteristic abrasive behavior," Precision Engineering, vol. 24, no. 3, pp. 237-244, 2000.

[13] Y. Wang and D. Hu, "Study on the inner surface finishing of tubing by magnetic abrasive finishing," International Journal of Machine Tools and Manufacture, vol. 45, no. 1, pp. 43-49, 2005.

[14] H. Yamaguchi and T. Shinmura, "Internal finishing process for alumina ceramic components by a magnetic field assisted finishing process," Precision Engineering, vol. 28, no. 2, pp. 135-142, 2004.

[15] S. Singh and H. S. Shan, "Development of magneto abrasive flow machining process," International Journal of Machine Tools and Manufacture, vol. 42, no. 8, pp. 953-959, 2002.

[16] J. D. Kim, D. X. Jin, and M. S. Choi, "Study on the effect of a magnetic field on an electrolytic finishing process," International Journal of Machine Tools and Manufacture, vol. 37, no. 4, pp. 401-408, 1997.

[17] H. E. D. Brujin, T. H. Delft, and A. J. Pekelhang, "Effect of magnetic field on the gap cleaning in EDM," Annals of the CIRP, vol. 27, no. 1, pp. 93-95, 1978.

[18] Y. C. Lin and H. S. Lee, "Machining characteristics of magnetic force-assisted EDM," International Journal of Machine Tools and Manufacture, vol. 48, no. 11, pp. 1179-1186, 2008.

[19] K. D. Chattopadhyay, P. S. Satsangi, S. Verma, and P. C. Sharma, "Analysis of rotary electrical discharge machining characteristics in reversal magnetic field for copper-en8 steel system," International Journal of Advanced Manufacturing Technology, vol. 38, no. 9-10, pp. 925-937, 2008. 

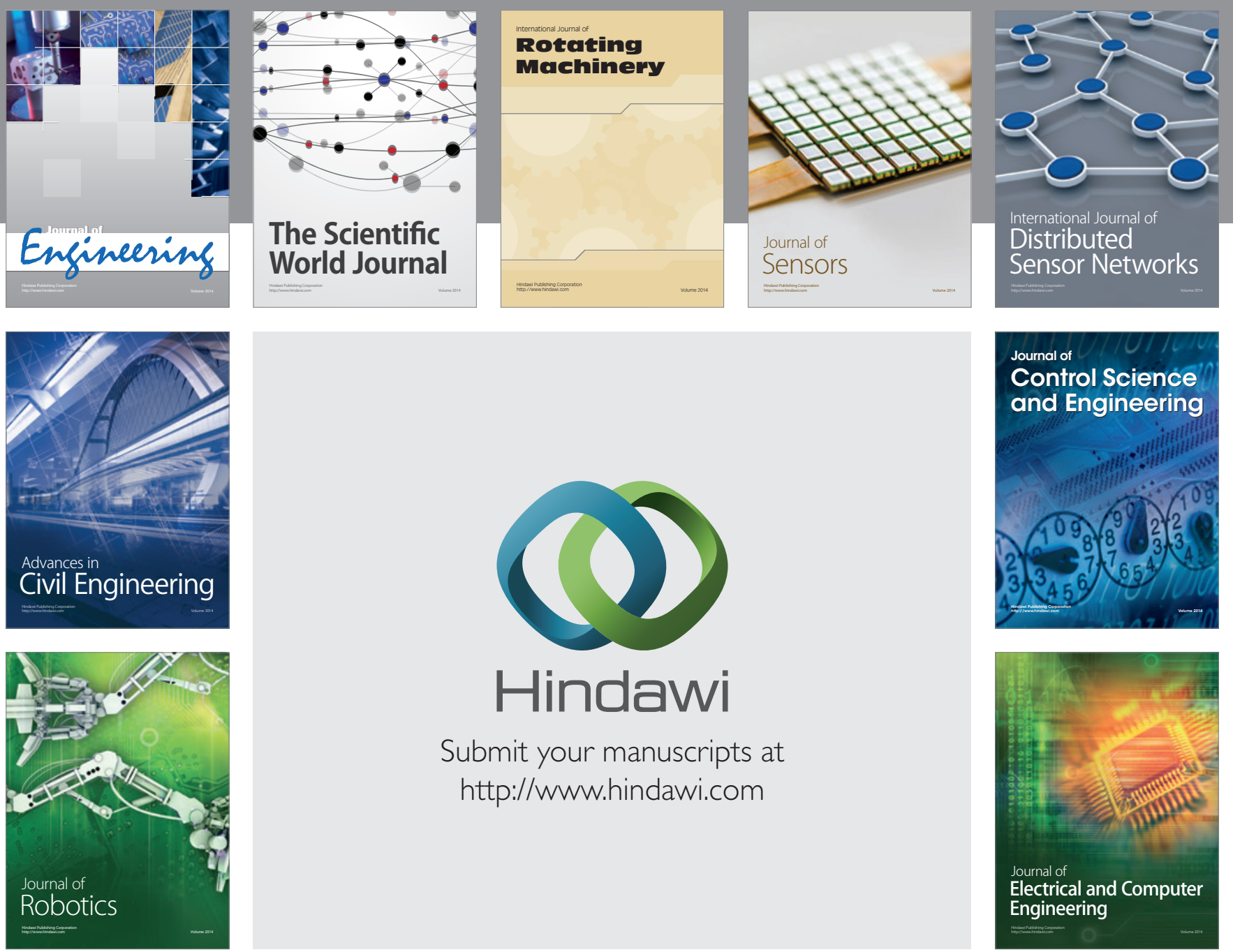

Submit your manuscripts at

http://www.hindawi.com
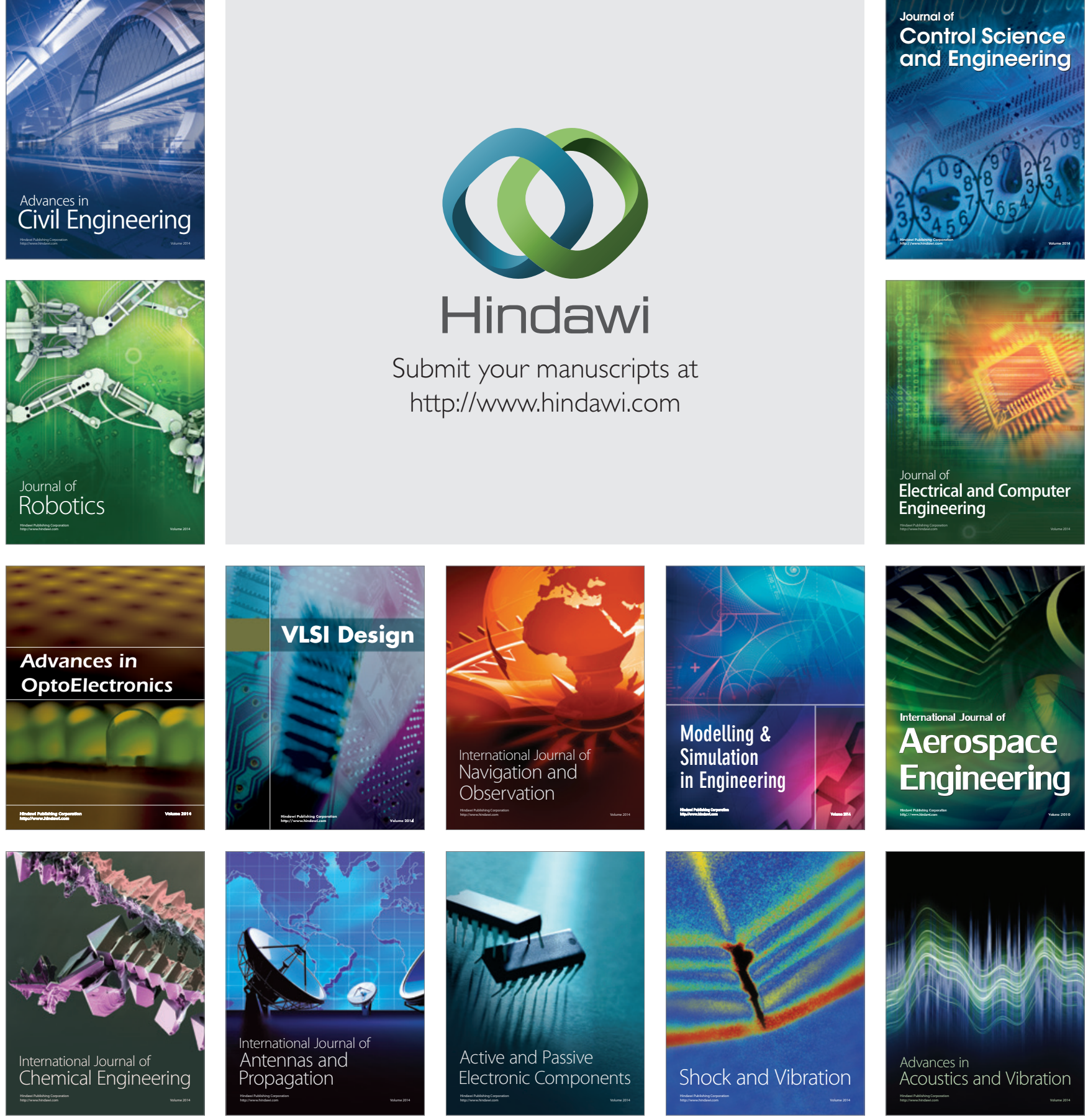